\title{
PESO DETERMINA O RESULTADO DE DISPUTAS TERRITORIAIS EM ERYTHRODIPLAX FUSCA RAMBUR, 1842 (ODONATA: LibeLLULIDAE)
}

\author{
Deborah Costa Natividade
}

\author{
Querem Cristine Ferreira Santana
}

\author{
Nelson Silva Pinto \\ Centro Universitário Araguaia, Av. T-10, 1047 - Setor Bueno, Goiânia, 74223-060, \\ Goiás, Brasil, nelsonsilvapinto@gmail.com
}

\begin{abstract}
Resumo: Erythrodiplax fusca (Rambur, 1842) é uma Odonata da família Libellulidae, que defendem seus territórios próxima de rios, lagos e áreas pântanosas. Disputas por acesso a fêmeas ou pelos locais de reprodução, geralmente ocorrem durante o voo. O objetivo neste trabalho foi analisar as disputas por territórios entre machos avaliar se o ganhador possuía maior peso. O estudo foi conduzido na Fazenda Experimental Morro Feio, localizada no município de Hidrolândia, estado de Goiás, Brasil. Inicialmente, foi realizada uma inspeção para encontrar pontos na margem do riacho que os machos comumente disputam para estabelecer seus territórios. Após a identificação destes pontos todas as disputas por território foram contabilizadas, ao final do conflito os dois machos foram coletados (vencedor e perdedor) e pesados. Foram coletados 74 indivíduos de E. fusca (37 vencedores e 37 perdedores). O peso fresco foi determinante para a vitória, com machos vitoriosos apresentando peso fresco $10 \%$ maior que os machos perdedores. Portanto, o presente trabalho corrobora a hipótese de que o peso dos machos de E. fusca está relacionado à vitória em disputas por territórios.
\end{abstract}

Palavras-chaves: conflito, peso, RHP, disputas e território.

\section{WEIGHT DETERMINES THE OUTCOME IN TERRITORIAL CONTESTS OF ERYTHRODIPLAX FUSCA RAMBUR, 1842 (ODONATA: LibELLULIDAE)}

\begin{abstract}
Erythrodiplax fusca (Rambur, 1842) is an Odonata of the Libellulidae family, which defend their territories close to rivers, lakes and swamps. Disputes over access to females or breeding sites usually occur during flight activities. The objective in this work was to analyze the disputes over territories between males and to evaluate if the winners are heavier than losers. The study was conducted at the Morro Feio Experimental Farm, located in the municipality of Hidrolândia, state of Goiás, Brazil. First, an inspection was carried out to find points on the bank of the stream that males commonly dispute to establish their territories. After the identification of these points, all disputes for territory were counted and at the end of the conflict the two males were collected (winner and loser) and weighed. 74 individuals of E. fusca (37 winners and 37 losers) were collected. The fresh weight was decisive to win the dispute, with winner males $10 \%$ heavier than losing males. Therefore, this work corroborates the hypothesis that the weight of the males of $E$. fusca is related to increases in probability of victory in disputes over territories.
\end{abstract}

Keywords: conflict, weight, RHP, contests and territory. 


\section{INTRODUÇÃO}

Animais disputam o acesso a recursos escassos e indivisíveis ao longo de seu ciclo de vida (Briffa \& Hardy, 2013). Esses recursos podem ser por exemplo, alimento, abrigo ou recursos que aumentem a probabilidade de acesso a parceiros reprodutivos (Briffa \& Hardy, 2013). Como essas disputas envolvem gastos energéticos altos que podem elevar riscos de injúria e predação, é possível que existam regras de resolução destas disputas. Uma destas regras na resolução desses conflitos está baseada na capacidade de luta dos indivíduos, chamada em inglês de resource holding power (RHP) com a vitória ficando para o indivíduo com maior capacidade de luta no momento da disputa (Parker, 1974).

O RHP pode ser determinado por fatores fisiológicos ou morfológicos (Arnott \& Elwood, 2009). Exemplos de características morfológicas e fisiológicas importantes para a resolução das disputas em diferentes espécies de animais podem ser o peso, a idade e o comprimento do corpo (e.g. Riechert, 1978; Forsyth \& Montgomerie, 1987; Draud \& Lynch, 2002; Resende, 2010; Junior \& Peixoto, 2013). A modificação nesses fatores entre diferentes indivíduos de uma população pode fazer com que eles obtenham resultados desiguais em relação à conquista de recursos.

As libélulas (Insecta: Odonata) são bons modelos para estudar regras de disputa por acesso a parceiros sexuais (e.g. Lefevre \& Muehter, 2004; Switzer, 2004; Junior \& Peixoto, 2013). Muitas espécies de libélulas são territoriais e disputam locais próximos aos corpos d'água que são periodicamente visitados por fêmeas (Corbet, 1980). Nessas espécies, as disputas por acesso a fêmeas ou pelos locais de reprodução, geralmente ocorrem durante o voo e essas interações agonísticas podem ocorrer com ou sem contato físico e ocorrência de ferimentos, frequentemente demorando de alguns segundos até minutos (Pinto et al., 2013).

Nas disputas de libélulas, tanto a quantidade de músculos (variável possivelmente relacionada à força e habilidade de voo) e a gordura corporal (variável possivelmente relacionada à persistência e quantidade de energia em reservas) podem estar relacionadas com a resolução de conflitos em espécies de libélulas (Junior \& Peixoto, 2013). Como essas duas variáveis podem ser acessadas indiretamente por medidas de peso fresco, pode-se esperar que o peso fresco do indivíduo esteja relacionado com a capacidade de luta (RHP).

A espécie Erythrodiplax fusca (Rambur, 1842) se destaca por ser um inseto que apresenta alta abundância em corpos d'água e comportamento territorialista (Resende, 2010). A espécie faz parte do grupo connata, que possui a fronte e tórax avermelhados e seu abdome pode ser avermelhado ou azulado (Borror, 1942). Os machos de E. fusca, defendem seus territórios em pontos distribuídos ao redor de lagos, rios e pântanos, eles empoleiram-se em galhos, macrófitas, gramíneas ou até mesmo no solo e as fêmeas depositam seus ovos diretamente na água, sobre vegetação submersa (De Marco et al., 2005; Resende \& De Marco, 2008). E. fusca é um Libellulidae de porte pequeno, com distribuição na região neotropical. É caracterizado como pousador (passa a maior parte de seu período de atividade pousado ou realizando voos curtos de inspeção próximos ao território), habita geralmente ambientes lênticos e sofre influência da temperatura sobre seu período de atividade (De Marco \& Resende, 2002). As fêmeas ficam dispersas, se alimentando, e retornam para esses pontos somente para copular e ovipositar (Resende, 2010).

Dado que não há evidências de quais variáveis determinam a probabilidade de vitória de machos de E. fusca em disputas por poleiros, neste trabalho, avaliamos se o peso fresco dos machos vivos está relacionado com a capacidade de luta de indivíduos de E. fusca. Para isso, testamos a hipótese de que os vencedores de disputas por poleiros em E. fusca apresentariam maior peso fresco quando comparados com os perdedores.

\section{Materiais e MÉtodos}

\section{ÁreA DE ESTUdo}

Este estudo foi conduzido na Fazenda Experimental Morro Feio, que corresponde a uma área de aproximadamente 100 ha, e está localizada no município de Hidrolândia, estado de Goiás, Brasil (Fig. 1). A fazenda apresenta áreas que contém mata de galeria típicas de Cerrado e possui duas estações predominantes, uma chuvosa que varia entre outubro-março e uma seca entre abril-setembro (Klink \& Machado, 2005). O local de estudo foi em trechos lênticos do córrego que banha a fazenda, sempre em trechos com pouca vegetação ciliar e alta incidência solar. A escolha destes locais deve-se ao fato de que a espécie modelo do estudo apresenta maior abundância nestes pontos (obs. pessoal). As observações comportamentais foram realizadas entre as 10:00 e 15:00 horas, duas vezes na semana no mês de outubro de 2019, totalizando 8 dias de coleta e 40 horas de observação. As coletas foram realizadas sempre com incidência solar direta e temperatura acima de $20^{\circ} \mathrm{C}$, pois é o período em que elas apresentam maior atividade (De Marco \& Resende, 2002). 


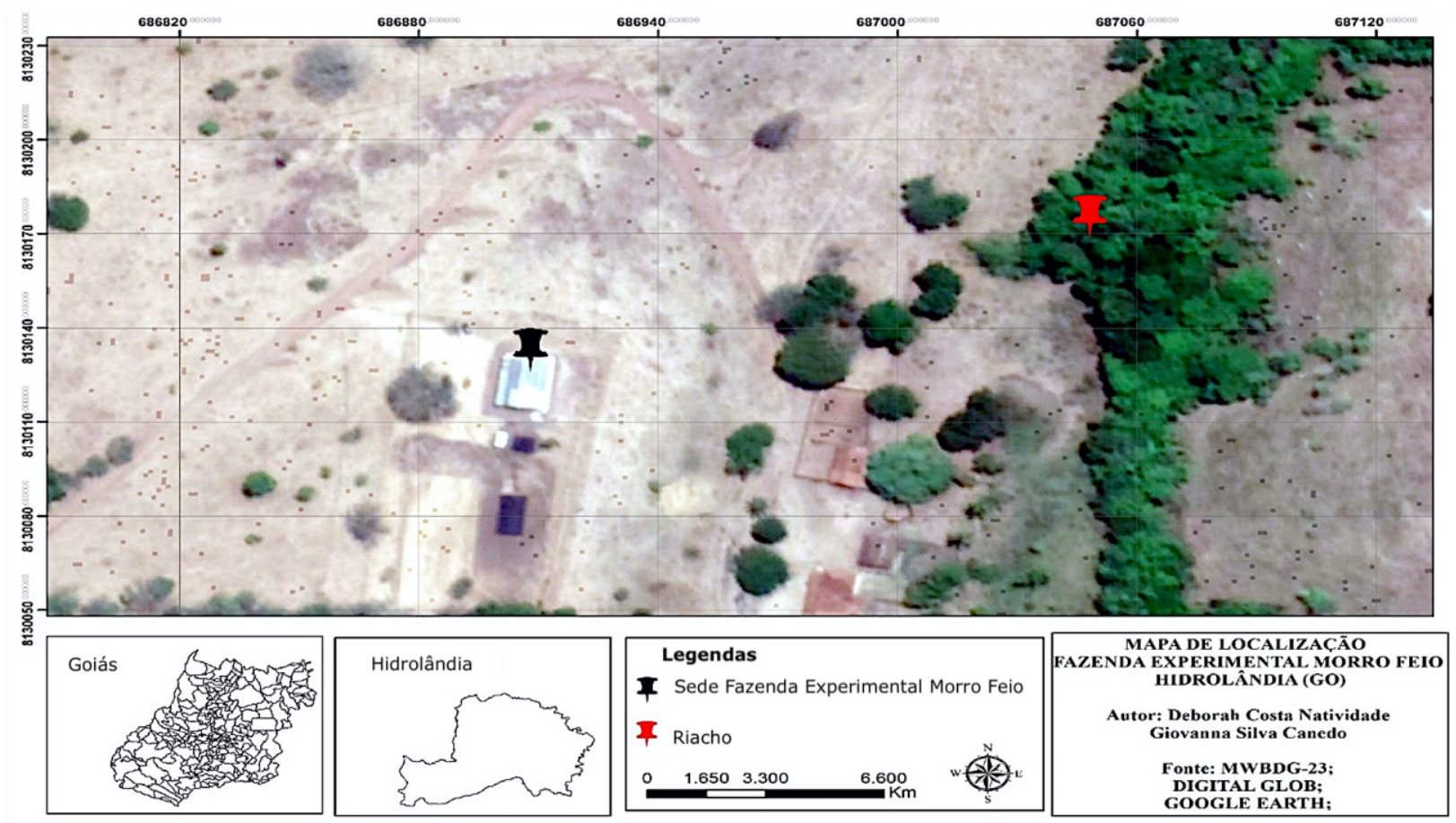

Fig. 1. Mapa de localização da Fazenda Morro Feio, município de Hidrolândia, estado de Goiás, Brasil. Fonte: MWDG-23; Digital Glob, Google Earth.

\section{Procedimentos experimentais}

No primeiro momento, realizamos uma inspeção para encontrar pontos nas margens em que os machos comumente disputam para estabelecer seus territórios. Geralmente, os machos de E. fusca usam pequenos galhos ou gramíneas próximas à margem como territórios, o ponto para o qual sempre retornam após uma interação agonística ou tentativa de cópula (Resende, 2010). Em seguida realizamos uma nova inspeção para coleta com a rede entomológica e marcação nas asas com tinta atóxica para facilitar a identificação dos indivíduos presentes nos poleiros. Os poleiros estavam em ambas as margens e com distâncias de aproximadamente 5 metros, com intuito de indivíduos próximos não serem influenciados.

Para avaliar a hipótese de que os indivíduos vencedores apresentariam maior peso fresco foi realizado o seguinte experimento. Todas as disputas por território nas quais os donos estiveram envolvidas foram acompanhadas. Após o final das disputas, os indivíduos invasores (i.e. o indivíduos que não possuem território e que tentam expulsar um dono para se estabelecer) e os indivíduos donos de territórios foram coletados. Foram atribuídos os status de perdedor e vencedor conforme o resultado de cada disputa. Ao todo, foram coletados 74 indivíduos (37 vencedores e 37 perdedores) com auxílio da rede entomológica, e todos eles foram pesados em balança de precisão $(0,001 \mathrm{~g}$, marca AVB, modelo FB). Os indivíduos observados foram co- letados apenas uma vez e o mesmo indivíduo não foi avaliado em disputas subsequentes, totalizando 37 disputas diferentes.

Para avaliar se havia diferença do peso fresco entre os indivíduos perdedores e vencedores, foi realizado um teste $t$ para amostras independentes, no qual a variável categórica foi o Status (Perdedor e Vencedor) e a variável contínua foi o peso fresco em gramas (g). Para avaliar a hipótese de que o peso fresco estaria relacionado com o aumento na probabilidade de vitória dos indivíduos, usamos uma regressão logística simples. Nesta análise, a variável resposta foi o status do indivíduo após a disputa e a variável preditora quantitativa foi o peso fresco em gramas ( $\mathrm{g}$ ). Usamos o software R para realizar as análises ( $R$ Development Core Team, 2015).

\section{Resultados}

\section{PadRões gerais de comportamento}

Foram observados 37 disputas entre machos donos de territórios e invasores. As interações se iniciavam com a chegada do macho invasor no território de seu adversário. Após essa fase, os machos vencedores realizavam um voo direto para cima e pouco tempo depois voltavam para seus territórios e os indivíduos que perderam se afastavam do local e voavam em busca de novas interações. 


\section{EFEITO dO PESO FRESCO NA RESOLUÇÃO DAS DISPU-} TAS

Todos os indivíduos envolvidos nas disputas foram coletados, totalizando 74 exemplares de $E$. fusca. Das interações observadas, 28 se atribuiu a vitória para machos que possuíam maior peso fresco em relação ao seu oponente. Os machos vencedores de modo geral possuíam maior peso fresco quando comparado com os machos que perderam as disputas $(\mathrm{t}=2,08 ; \mathrm{gl}$ $=72 ; p=0,042$, Fig. 2). Os machos vencedo- res tiveram peso fresco médio de $0,102 \mathrm{~g} \pm$ $0,022 \mathrm{~g}$ (média \pm desvio padrão) e os machos perdedores apresentaram peso fresco médio de $0,092 \mathrm{~g} \pm 0,019 \mathrm{~g}$. A diferença média entre 0 peso fresco de vencedores e perdedores foi de aproximadamente $0,01 \mathrm{~g}$ ou $10 \%$ do peso fresco médio dos indivíduos. A probabilidade de vitória aumentou com o aumento do peso fresco dos machos $\left(x^{2}=4,353, g l=1, p=0,037\right.$, Fig. 3$)$. A razão de probabilidades (odds ratio) foi igual a 0,083 .

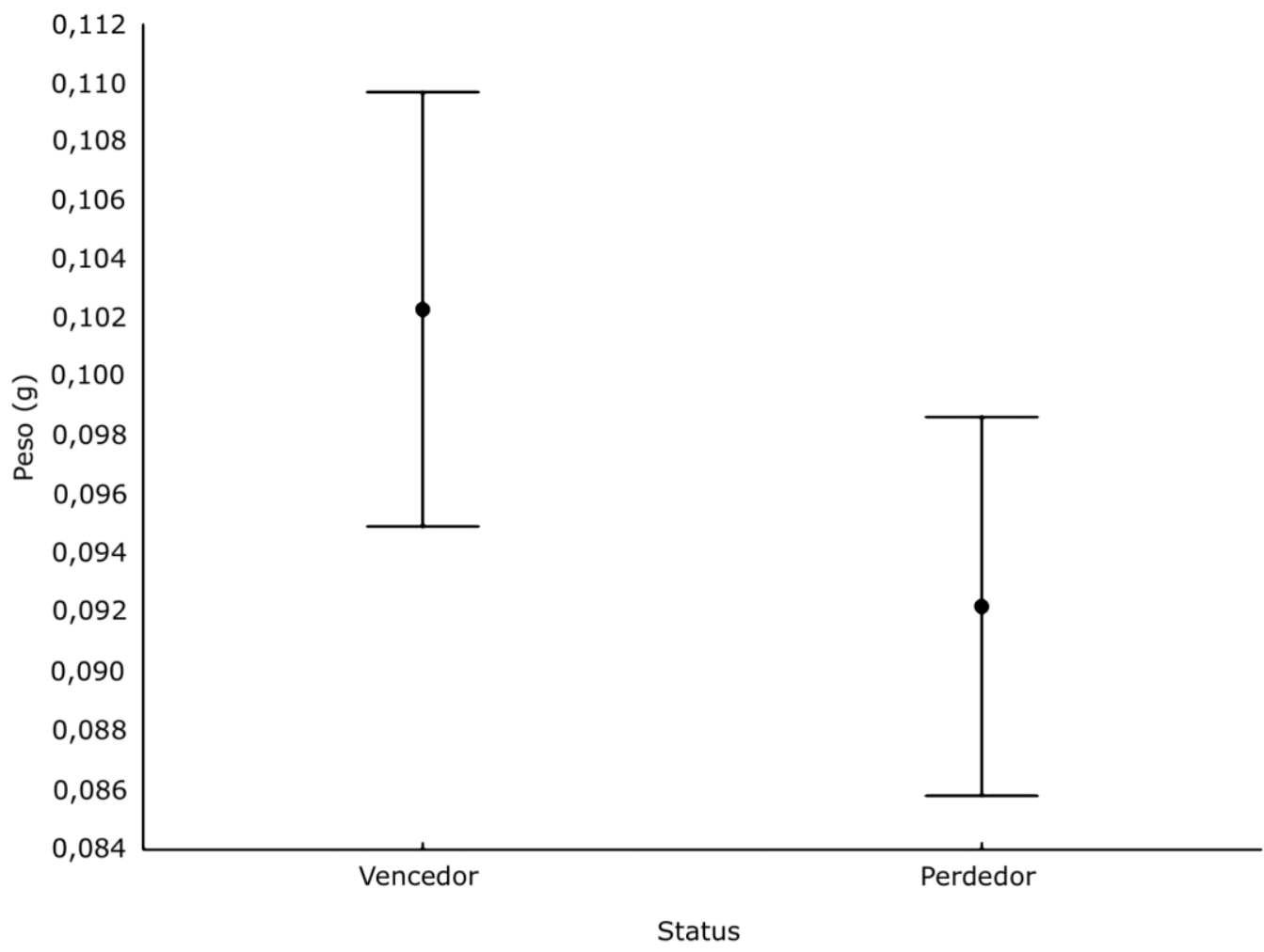

Fig. 2. Comparação das médias de peso in vivo entre as categorias vencedor e perdedor de indivíduos de Erythrodiplax fusca (Rambur, 1842), Hidrolândia, Goiás, Brasil. As barras denotam um intervalo de confiança de $95 \%$ e os pontos indicam os valores médios.

\section{Discussão}

Os machos de E. fusca realizam disputas para terem acesso a territórios, e em sua maioria, estas disputas ocorrem sem contato físico. Foi analisado também que, no caso dos machos de E. fusca observados na Fazenda Experimental Morro Feio, existe a presença de machos concorrendo e, consequentemente, ocorrem disputas por territórios. Nessas disputas observadas, o peso fresco está associado à probabilidade de vitória.

Observamos disputas com presença e au- sência de contato físico entre os indivíduos de E. fusca. O padrão de resolução de disputas com contato físico pode indicar que o uso de contato físico com injúria deve ocorrer apenas em situações extremas, como no caso de machos com comprimento corporal e/ou peso semelhante (Vieira \& Peixoto, 2013; Briffa \& Hardy, 2013). Geralmente nessas situações, os machos podem ser parecidos em relação ao tamanho corporal ou peso e, portanto, ter maior força e também maior quantidade de reservas de energia para investir nas disputas (Briffa \& Hardy, 2013; Junior \& Peixoto, 2013). No caso das libélulas aqui estudadas, a diferença do pe- 


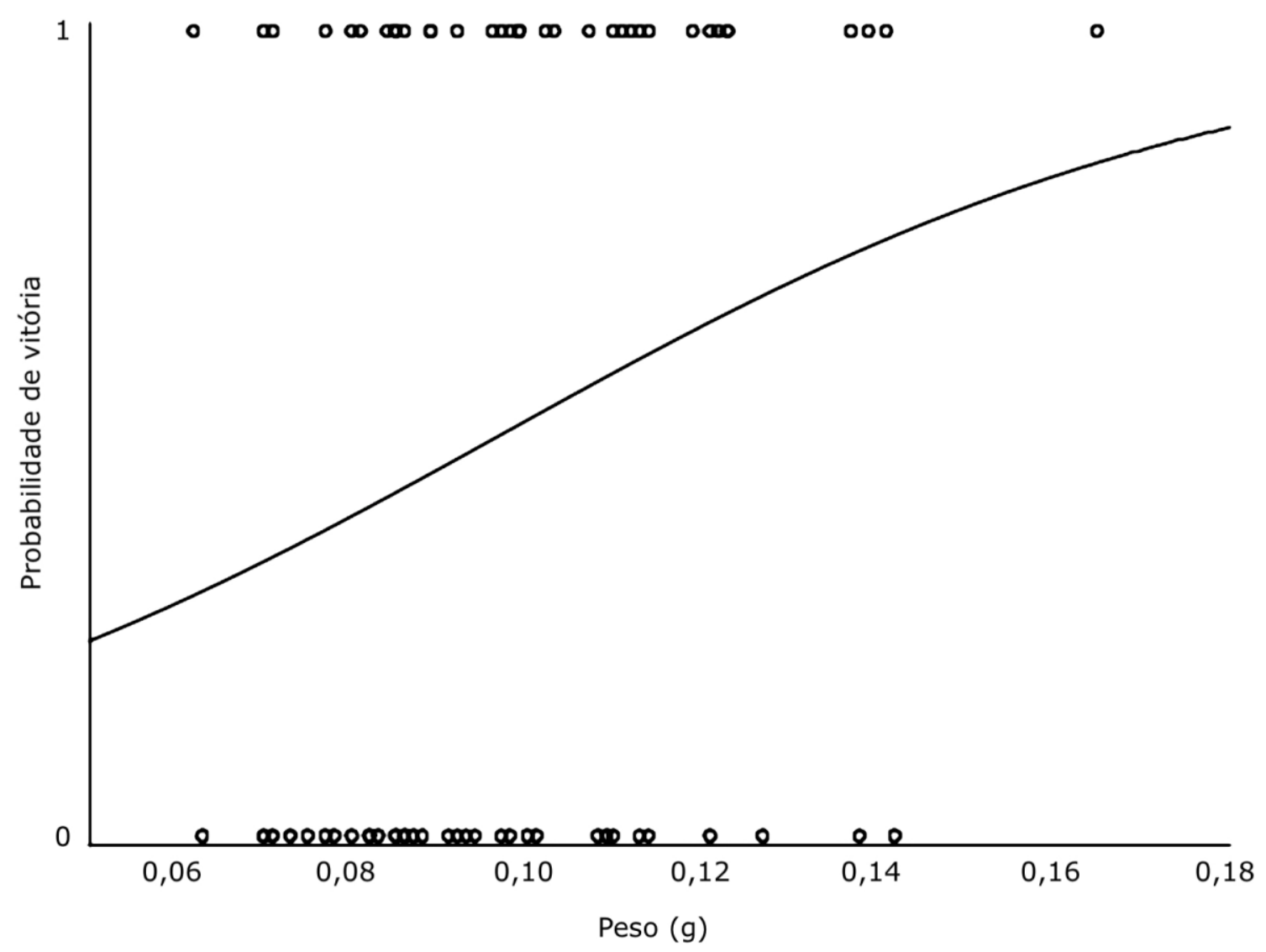

Fig. 3. Probabilidade de vitória dos indivíduos de Erythrodiplax fusca (Rambur, 1842), Hidrolândia, Goiás, Brasil em relação ao peso. No eixo $X$ observa-se o peso fresco dos indivíduos em gramas $(\mathrm{g})$. No eixo Y observa-se a probabilidade de vitória.

so fresco dos machos de E. fusca vencedores e perdedores pode ser suficiente para uma maior probabilidade de disputas sem contato físico e, consequentemente, de menor custo.

Os resultados sobre a relação do status e o peso fresco indicaram que machos mais pesados tem maior capacidade de luta (RHP), dado que machos vencedores apresentaram, em média, maior peso fresco que machos perdedores. Como ocorreram casos nos quais invasores maiores conseguiram vencer os donos já estabelecidos, é possível que o peso fresco maior seja mais importante do que o status de dono do território. Isto exclui ou ao menos diminui os efeitos relacionados a assimetrias não correlacionadas ao RHP, como a vantagem do residente (Switzer, 2004). Resultados parecidos indicando a prevalência de machos maiores foram observados para grilos Hemideina crassidens (Blanchard, 1851) (Kelly, 2006), borboletas Paryphthimoides phronius (Godart, 1824) (Peixoto \& Benson, 2008) e aranhas Phidippus clarus (Keyserling, 1885) (Elias et al., 2008). Estes resultados corroboram a importância da capacidade de luta para a resolução de disputas, indi- cada em uma meta-análise recente (Vieira \& Peixoto 2013).

Existe uma diferença de peso fresco entre os machos que venceram as disputas (machos que permaneceram no território) em relação aos machos que perderam (machos que buscaram novos territórios). Dessa forma, este trabaIho corroborou a hipótese de que o peso fresco dos machos de $E$. fusca está relacionado à probabilidade de vitória em disputas por territórios. Estes resultados são importantes para pesquisas futuras relacionadas ao comportamento de Odonata, principalmente análises envolvendo interações agonísticas.

\section{Agradecimentos}

Este manuscrito é parte dos resultados do projeto: Estudo da Biodiversidade da Fauna e Flora da Fazenda Experimental Morro Feio (Hidrolândia-GO, Brasil) como Estratégia para Educação Ambiental, do Centro Universitário Araguaia. NSP agradece ao CNPq pela concessão de bolsa de pós-doutorado (CNPq-Processo: 
160656/2019-2). Agradecemos também aos revisores pelas sugestões que contribuíram muito para a melhoria do manuscrito.

\section{REFERÊNCIAS}

Arnott, G. \& R. W. Elwood. 2009. Assessment of fighting ability in animal contests. Anim. Behav. 77: 991-1004. DOI: https://doi.org/ 10.1016/j.anbehav.2009.02.010

Borror. D. 1942. A revision of the Libelluline Genus Erythrodiplax (Odonata), Ohio, Ohio State University.

Briffa, M. \& I. C. W. Hardy. 2013. Introduction to animal contests. pp. 1-4. In: Hardy, I. C. W. \& M. Briffa. Animal Contests. New York, Cambridge University press.

Corbet, P. S. 1980. Biology of Odonata. Annu. Rev. Entomol. 25: 189-217. DOI: https:// doi.org/10.1146/annurev.en. 25.010180 .001201

De Marco, P., A. O. Latini, \& D. C. Resende. 2005. Thermoregulatory constraints on behavior: patterns in a neotropical dragonfly assemblage. Neotrop. Entomol. 34: 155-162. DOI: https://doi.org/10.1590/ S1519-566X2005000200002

De Marco. P. \& D. C. Resende. 2002. Activity patterns and thermoregulation in a tropical dragonfly assemblage. Odonatologica. 31: 129-139.

Draud, M. \& P. A. E. Lynch. 2002. Asymmetric contests for breeding sites between monogamous pairs of convict cichlids (Archocentrus nigrofasciatum, (ichlidae): pair experience pays. Anim. Behav. 139: 861873.

Elias, D. O., M. M. Kasumovic, D. Punzalan, M. C. B. Andrade \& A. C. Mason. 2008. Assessment during aggressive contests between male jumping spiders. Anim. Behav. 76: 901-910.

Forsyth, A. \& R. D. Montgomerie. 1987. Alternative reproductive tactics in the territorial damselfly Calopteryx maculata: sneaking by older males. Behav. Ecol. Sociobiol. 21: 73-81. DOI: https://doi.org/ 10.1007/BF02395434
Junior, R. S. L. \& P. E. C. Peixoto. 2013. Males of the dragonfly Diastatops obscura fight according to predictions from game theory models. Anim. Behav. 85: 663-669. DOI: https://doi.org/10.1016/j.anbehav. 2012.12.033

Kelly, C. D. 2006. Fighting for harems: assessment strategies during male-male contests in the sexually dimorphic Wellington tree weta. Anim. Behav. 72: 727-736.

Klink, C. A. \& R. Machado. 2005. A conservação do Cerrado brasileiro. Megadiversidade. 1: 147-155.

Lefevre, K. L. \& V. R. Muehter. 2004. Competition for mating resources in a territorial damselfly (Odonata: Calopterygidae). Stud. Neotrop. Fauna Environ. 39: 159165.

Parker, G. A. 1974. Assessment strategy and the evolution of fighting behaviour. J. Theor. Biol. 47: 223-243. DOI: https://doi.org/ 10.1016/0022-5193(74)90111-8

Peixoto, P. E. C. \& W. M. Benson. 2008. Body Mass and not Wing Length Predicts Territorial Success in a Tropical Satyrine Butterfly. Ethology. 114: 1069-1077. DOI: https:// doi.org/10.1111/j.14390310.2008.01555.x

Pinto, N. S., J. Hidasi-Neto, V. Ribeiro, A. R. Rodrigues, B. R. Brandão \& C. O. Rocha. 2013. Efeito da Presença de Vizinhos sobre o Comportamento Territorial de Perithemis mooma (Kirby) (Anisoptera: Libellulidae). EntomoBrasilis. 6: 104-107. DOI: https://doi.org/10.12741/ebrasilis.v6i2.285

R Development Core Team. 2015. R: A Language and Environment for Statistical Computing. R Foundation for Statistical Computing, Vienna Electronic file available at http://www.R-project.org.

Resende, D. C. 2010. Residence advantage in heterospecific territorial disputes of Erythrodiplax Brauer species (Odonata, Libellulidae). Rev. Bras. Entomol. 54: 110-114. DOI: $\quad$ https://doi.org/10.1590/S008556262010000100014 
Resende, D. \& P. De Marco. 2008. Residence and territorial characteristics of Libellulidae species in a neotropical assemblage (Anisoptera). Odonatologica. 37: 213-220.

Riechert, S. E. 1978. Games spiders play: behavioral variability in territorial disputes. Behav. Ecol. Sociobiol. 3: 135-162. DOI: https://doi.org/10.1007/BF00294986

Switzer, P. V. 2004. Fighting behavior and prior residency advantage in the territorial dragonfly, Perithemis tenera. Ethol. Ecol. Evol., 16: 71-89. DOI: https://doi.org/ 10.1080/08927014.2004.9522655

Vieira, M. C. \& P. E. C. Peixoto. 2013. Winners and losers: a meta-analysis of functional determinants of fighting ability in arthropod contests. Func. Ecol. 27: 305313. DOI: https://doi.org/10.1111/13652435.12051 